\title{
Anticipation and the control of voluntary action
}

\section{Dorit Wenke $^{1 *}$ and Rico Fischer ${ }^{2 *}$}

1 Department of Psychology, Humboldt-Universität zu Berlin, Berlin, Germany

${ }^{2}$ Department of Psychology, Technische Universität Dresden, Dresden, Germany

*Correspondence: dorit.wenke@hu-berlin.de; fischer@psychologie.tu-dresden.de

Edited by:

Bernhard Hommel, Leiden University, Netherlands

A major hallmark in the adaptive control of voluntary action is the ability to anticipate short and long term future events. Anticipation in its various forms is an important prerequisite for cognitive abilities such as planning, reasoning and the pursuit of both immediate goals and long-term goals (e.g., to invest in pension funds) that sometimes stand in opposition to immediate desires and needs. Therefore, it is not surprising that diverse and rather independent research lines have evolved, all somehow targeting various anticipatory capacities that are involved in the control of voluntary action.

One line of research focuses on anticipating action effects. For example, ideomotor theory assumes that actions are selected and activated by the mere anticipation of the sensory experience they produce (James, 1890/1950). Similarly, prediction of the incentive value of action outcomes has been proposed to drive goaldirected instrumental behavior (Balleine and Dickinson, 1998). Furthermore, the degree of match between intended, anticipated and actual action effects seems to be a major determinant of motor programming and online corrections (Prablanc and Martin, 1992), motor learning (Wolpert et al., 2011), and the subjective sense of causing and controlling actions and their effects (the Sense of Agency; Frith et al., 2000). However, the role of anticipation in the control of voluntary action goes beyond the anticipation of action effects. For instance, pre-cues and alerting signals are used for preparing what to do (Meiran, 1996), when to act or expect an event, (Callejas et al., 2004) and for anticipating conflict (Correa et al., 2009). Similarly, learning of statistical contingencies leads to prediction of context-specific executive control requirements (Crump et al., 2006).

The aim of the present Research Topic has been to provide a platform that offers the possibility of cross-fertilization and enhanced visibility among to date rather segregated research lines concerning the role of anticipation in the control of voluntary action.

Many contributions address the role of anticipating action effects in controlling and understanding actions. Some deal with the role of anticipated value of action outcomes: Watson et al. (2012) provide a review on maladaptive drug seeking behavior from a learning theory perspective. Pezzulo et al. (2013) propose a model in which a single mixed controller balances habitual choice based on cached action values, and mental simulations of action outcomes that underlie goal directed behavior, depending on the usefulness of obtaining new information. Scherbaum et al. (2012) propose a model of temporal discounting - the tendency to choose smaller rewards delivered sooner instead of larger rewards delivered later- that focuses on response threshold and time framing as two factors determining choice behavior in inter-temporal choice.

Further contributions address the role of effect anticipation and feedback evaluation in the control and experience of action: Schilling and Cruse (2012) propose a predictive body model for planning robots' actions. Wang et al. (2012) present data showing that distorted visual movement feedback tends to affect action evaluation more strongly in old than in young adults. Haering and Kiesel (2012) demonstrate that prior causal beliefs influence intentional binding, a temporal illusion often seen as an indirect measure of sense of agency. Hommel and Keizer (2012) show that that object files can contain evaluative information regarding the match (success) viz. mismatch (failure) between predicted and experienced events. Poehlman et al. (2012) argue that supramodal integration through conscious states is primarily related to the skeletal muscle output system where anticipatory processes play a central role.

Thinnes-Elker et al. (2012) discuss different concepts of intention with respect to their implications for brain-machineinterfaces that "decode" brain activity for controlling artificial effectors. Because anticipating the consequences of one's own and others actions is an important aspect of social interactions and sport settings, Weigelt and Memmert (2012) investigated how the implicit processing of the stimulus layout in natural scenes affects the goal-side selection in soccer penalty shooting.

Predictive mechanisms are also involved in our ability to understand other people's actions, and even infants tend to interpret various action components with respect to action goals. In this line, Daum et al. (2012) demonstrate a dissociation between two measures often used to investigate expectations about goaldirected actions in infants, namely post-hoc looking times and predictive gaze. Henrichs et al. (2012) report evidence for an impact of goal salience on infants' goal anticipations of observed reaching actions, as measured by predictive gaze.

Another group of contributions focusses on the role of implicit or explicit cues that are utilized by the cognitive system to adjust cognitive control: Wendt et al. (2012) show that cue-based task preparation during task-switching is modulated by the validity of preceding trial task-cues. Strack et al. (2013) investigated cue-induced preparation, aiming at disentangling anticipatory control adjustments and prevention of upcoming conflict via task recoding. King et al. (2012) applied a model-based analysis and argue that context-specific proportion congruence effects may be accounted for by a prediction error-triggered shift in the decision criterion. Bugg and Crump (2012) provide a review on list-wide, item-specific and context-specific proportion congruence effects. 
Reuss et al. (2012) report data suggesting that participants can form expectations of where an event will occur on the basis of non-consciously presented cues. Duthoo et al. (2012) highlight the role of task repetition expectancy in task-switching by varying switch rate contingencies. Fröber and Dreisbach (2012) report data showing that positive affect with low arousal reduced proactive control as indicated by response cueing effects. Umbach et al. (2012) explored how explicit expectations feed into preparatory processes, over and above demand for preparation.

\section{REFERENCES ${ }^{1}$}

Balleine, B. W., and Dickinson, A. (1998). Goal-directed instrumental action: contingency and incentive learning and their cortical substrates. Neuropharmacology 37, 407-419.

*Bugg, J. M., and Crump, M. J. C. (2012). In support of a distinction between voluntary and stimulusdriven control: a review of the literature on proportion congruent effects. Front. Psychol. 3:367. doi: 10.3389/fpsyg.2012.00367

Callejas, A., Lupianez, J., and Tudela, P. (2004). The three attentional networks: on their independence and interactions. Brain Cogn. 54, 225-227. doi: 10.1016/j.bandc.2004.02.012

Correa, A., Rao, A., and Nobre, A. C. (2009). Anticipating conflict facilitates controlled stimulusresponse selection. J. Cogn. Neurosci. 21, 1461-1472. doi: 10.1162/jocn.2009.21136

Crump, M. J., Gong, Z., and Milliken, B. (2006). The context-specific proportion congruent Stroop effect: location as a contextual cue. Psychon. Bull. Rev. 13, 316-321.

*Daum, M. M., Attig, M., Gunawan, R., Prinz, W., and Gredebäck, G. (2012). Actions seen through babies' eyes: a dissociation between looking time and predictive gaze. Front. Psychol. 3:370. doi: 10.3389/fpsyg.2012.00370

* de la Rosa, M. D., Sanabria, D., Capizzi, M., and Correa, A. (2012). Temporal preparation driven by rhythms is resistant to working memory interference. Front. Psychol. 3:308. doi: 10.3389/fpsyg.2012.00308

*Duthoo, W., De Baene, W., Wühr, P., and Notebaert, W. (2012). When predictions take control: the effect of task predictions on task switching performance. Front. Psychol. 3:282. doi: 10.3389/fpsyg.2012.00282
Frith, C. D., Blakemore, S., and Wolpert, D. M. (2000). Explaining the symptoms of schizophrenia: abnormalities in the awareness of action. Brain Res. Rev. 31, 357-363.

*Fröber, K., and Dreisbach, G. (2012). How positive affect modulates proactive control: reduced usage of informative cues under positive affect with low arousal. Front. Psychol. 3:265. doi: 10.3389/fpsyg. 2012.00265

*Haering, C., and Kiesel, A. (2012). Mine is earlier than yours: causal beliefs influence the perceived time of action effects. Front. Psychol. 3:393. doi: 10.3389/fpsyg.2012.00393

*Henrichs, I., Elsner, C., Elsner, B., and Gredebäck, G. (2012). Goal salience affects infants' goal-directed gaze shifts. Front. Psychol. 3:391. doi: 10.3389/fpsyg.2012.00391

*Hommel, B., and Keizer, A. W. (2012). Binding success and failure: evidence for the spontaneous integration of perceptual features and object evaluations. Front. Psychol. 3:581. doi: 10.3389/fpsyg.2012.00581

James, W. (1890/1950). The Principles of Psychology. Vol. 2. New York, NY: Dover.

*King, J. A., Donkin, C., Korb, F. M., and Egner, T. (2012). Model-based analysis of context-specific cognitive control. Front. Psychol. 3:358. doi: 10.3389/fpsyg.2012.00358

Meiran, N. (1996). Reconfiguration of processing mode prior to task performance. J. Exp. Psychol. Learn. Mem. Cogn. 22, 1423-1442.

*Pezzulo, G., Rigoli, F., and Chersi, F. (2013). The mixed instrumental controller: using value of information to combine habitual choice and mental simulation. Front. Psychol. 4:92. doi: 10.3389/fpsyg. 2013.00092

*Poehlman, T. A., Jantz, T. K., and Morsella, E. (2012). Adaptive

A final group of contributions targets temporal anticipation in the control of voluntary action. Predicting the temporal onset of an event by means of a warning cue allows for temporal orienting and anticipation of an upcoming event. In a brief review, Weinbach and Henik (2012) discuss whether the temporal orienting function of warning cues can be dissociated from cue-based increases of alertness. Finally, de la Rosa et al. (2012) show that temporal preparation guided by regular rhythms is not subject to working memory interference and facilitates performance irrespective of concurrent working memory load.

skeletal muscle action requires anticipation and "conscious broadcasting". Front. Psychol. 3:369. doi: 10.3389/fpsyg.2012.00369

Prablanc, C., and Martin, O. (1992). Automatic control during hand reaching at undetected twodimensional target displacements. J. Neurophysiol. 67, 455-469.

*Reuss, H., Kiesel, A., Kunde, W., and Wühr, P. (2012). A cue from the unconscious - masked symbols prompt spatial anticipation. Front. Psychol. 3:397. doi: 10.3389/fpsyg.2012.00397

*Scherbaum, S., Dshemuchadse, M., and Goschke, T. (2012). Building a bridge into the future: dynamic connectionist modeling as an integrative tool for research on intertemporal choice. Front. Psychol. 3:514. doi: 10.3389/fpsyg.2012.00514

*Schilling, M., and Cruse, H. (2012). What's next: recruitment of a grounded predictive body model for planning a robot's actions. Front. Psychol. 3:383. doi: 10.3389/fpsyg.2012.00383

*Strack, G., Kaufmann, C., Kehrer, S., Brandt, S., and Stürmer, B. (2013). Anticipatory regulation of action control in a Simon task: behavioral, electrophysiological, and fMRI correlates. Front. Psychol. 4:47. doi: 10.3389/fpsyg.2013.00047

*Thinnes-Elker, F., Iljina, O., Apostolides, J. K., Kraemer, F., Schulze-Bonhage, A., and Aertsen, A. (2012). Intention concepts and brain-machine interfacing. Front. Psychol. 3:455. doi 10.3389/fpsyg.2012.00455

*Umbach, V. J., Schwager, S., Frensch, P. A., and Gaschler, R. (2012). Does explicit expectation really affect preparation? Front. Psychol. 3:378. doi: 10.3389 /fpsyg.2012.00378

*Wang, L., Sutter, C., Müsseler, J., Dangel, R. J. Z., and DisselhorstKlug, C. (2012). Perceiving one's own limb movements with conflicting sensory feedback: the role of mode of movement control and age. Front. Psychol. 3:289. doi: 10.3389/fpsyg.2012.00289

*Watson, P., de Wit, S., Hommel, B., and Wiers, R. W. (2012). Motivational mechanisms and outcome expectancies underlying the approach bias toward addictive substances. Front. Psychol. 3:440 doi: 10.3389/fpsyg.2012.00440

*Weigelt, M., and Memmert, D. (2012). Goal-side selection in soccer penalty kicking when viewing natural scenes. Front. Psychol. 3:312. doi: 10.3389/fpsyg.2012.00312

*Weinbach, N., and Henik, A. (2012). Temporal orienting and alerting - the same or different? Front. Psychol. 3:236. doi 10.3389/fpsyg.2012.00236

*Wendt, M., Luna-Rodriguez, A., Reisenauer, R., Jacobsen, T., and Dreisbach, G. (2012). Sequential modulation of cue use in the task switching paradigm. Front. Psychol. 3:287. doi: 10.3389/fpsyg.2012.00287

Wolpert, D. M., Diedrichsen, J., and Flanagan, J. R. (2011). Principles of sensorimotor learning. Nat. Rev. Neurosci. 12, 739-751.

Received: 24 May 2013; accepted: 24 May 2013; published online: 14 June 2013.

Citation: Wenke D and Fischer R (2013) Anticipation and the control of voluntary action. Front. Psychol. 4:341. doi: 10.3389/fpsyg.2013.00341

This article was submitted to Frontiers in Cognition, a specialty of Frontiers in Psychology.

Copyright (c) 2013 Wenke and Fischer. This is an open-access article distributed under the terms of the Creative Commons Attribution License, which permits use, distribution and reproduction in other forums, provided the original authors and source are credited and subject to any copyright notices concerning any third-party graphics etc.

\footnotetext{
${ }^{1}$ Articles that are part of the research topic are marked with asterisks $(*)$.
} 